\title{
El choque de estrellas de neutrones y la alquimia del Universo
}

\author{
Miguel Alcubierre Moya
}

\begin{abstract}
Resumen
El origen de los elementos ha sido uno de los más grandes misterios de la ciencia desde hace siglos. Los descubrimientos de la primera mitad del siglo XX nos permitieron entender la estructura de los átomos y de los núcleos atómicos, y nos llevaron al descubrimiento de que los elementos pesados se forman en el interior de las estrellas a partir de la fusión de elementos ligeros como el hidrógeno y el helio. Sin embargo, elementos más pesados que el hierro sólo se pueden formar en procesos cataclísmicos, como la explosión de una supernova, o el choque de dos estrellas. La observación reciente del choque de dos estrellas de neutrones, proceso conocido como kilonova, tanto en ondas gravitacionales como en todo el espectro electromagnético, nos ha permitido comprobar que este tipo de eventos producen elementos pesados, en particular metales preciosos en cantidades abundantes y a la vez los dispersan por el Universo.
\end{abstract}

Palabras clave: relatividad, gravitación, ondas gravitacionales, estrellas de neutrones.

\section{The COllision of neUtron stars and the alChEMy of the UNIVERSE}

\begin{abstract}
The origin of the elements has been one of the largest mysteries in science for centuries. The discoveries of the first half of the twentieth century allowed us to understand the structure of atoms and their nuclei, and led us to the discovery that the heavy elements can be formed inside stars from the fusion of lighter elements such as hydrogen and helium. However, elements heavier than iron can only be produced in cataclysmic processes, such as the explosion of a supernova, or the collision of two stars. The recent observation of the collision of two neutron stars, also known as a kilonova, both in gravitational waves and in the whole electromagnetic spectrum, has allowed us to verify that such events can produce heavy elements, and particularly precious metals in abundant quantities and at the same time help disperse them throughout the Universe.
\end{abstract}

Key words: relativity, gravity, gravitational waves, neutron stars.

DOI: http://doi.org/10.22201/codeic.16076079e.2018.v19n3.a3 


\section{Miguel Alcubierre Moya}

malcubi@nucleares.unam.mx

Estudió la licenciatura en Física en la Facultad de Ciencias de la Universidad Nacional Autónoma de México (UNAM), donde recibió la medalla Gabino Barreda. Obtuvo el grado de Maestro en Ciencias por la Facultad de Ciencias de la UNAM, y estudió el doctorado en la Universidad de Gales en Cardiff, Reino Unido, donde realizó una estancia posdoctoral. Trabajó durante varios años como investigador en el Instituto Max Planck para Física Gravitacional en Potsdam, Alemania. Es investigador titular en el Instituto de Ciencias Nucleares de la UNAM. Su área de investigación es la relatividad numérica, es decir, la simulación computacional de sistemas astrofísicos utilizando la teoría de la relatividad general de Einstein. Dentro de esta área se ha concentrado en la simulación de fuentes de ondas gravitacionales, particularmente la colisión de dos agujeros negros. Es autor de más de 50 publicaciones, así como de un libro de texto publicado por Oxford University Press. Es miembro del Sistema Nacional de Investigadores en el nivel más alto y de la Academia Mexicana de Ciencias.

\section{El origen de los elementos: la nucleosíntesis estelar}

Desde hace siglos filósofos y científicos se preguntaron sobre el origen y la posible trasmutación de los distintos elementos. Pero hubo que esperar hasta la llegada de la teoría atómica moderna a principios del siglo xx para obtener las primeras respuestas.

Con el descubrimiento de los electrones en 1897 por parte de Joseph John Thomson, y del núcleo atómico en 1911 por parte de Ernest Rutherford, la estructura básica del átomo quedó establecida: un núcleo compacto y masivo con carga eléctrica positiva, rodeado de electrones mucho más ligeros y con carga eléctrica negativa en órbita alrededor del núcleo. Las propiedades de las órbitas de los electrones fueron establecidas poco después por Niels Bohr en 1913, dando así inicio a la teoría cuántica moderna. En años posteriores, Rutherford mostró que el núcleo del átomo de hidrógeno, el más ligero de todos, estaba presente en el interior de los núcleos de los otros elementos, y en 1920 se le bautizó como "protón": una partícula de carga eléctrica positiva igual en magnitud a la del electrón, pero aproximadamente 2000 veces más masiva.

Para 1920, Arthur Eddington utilizó las ideas recientes sobre el modelo atómico, junto con la teoría de la relatividad de Albert Einstein, para intentar entender el origen de la energía del Sol y las estrellas. En esa época, la teoría prevaleciente, debida a Lord Kelvin entre otros, era que el Sol brillaba debido a la liberación de energía gravitacional al contraerse poco a poco durante millo- 


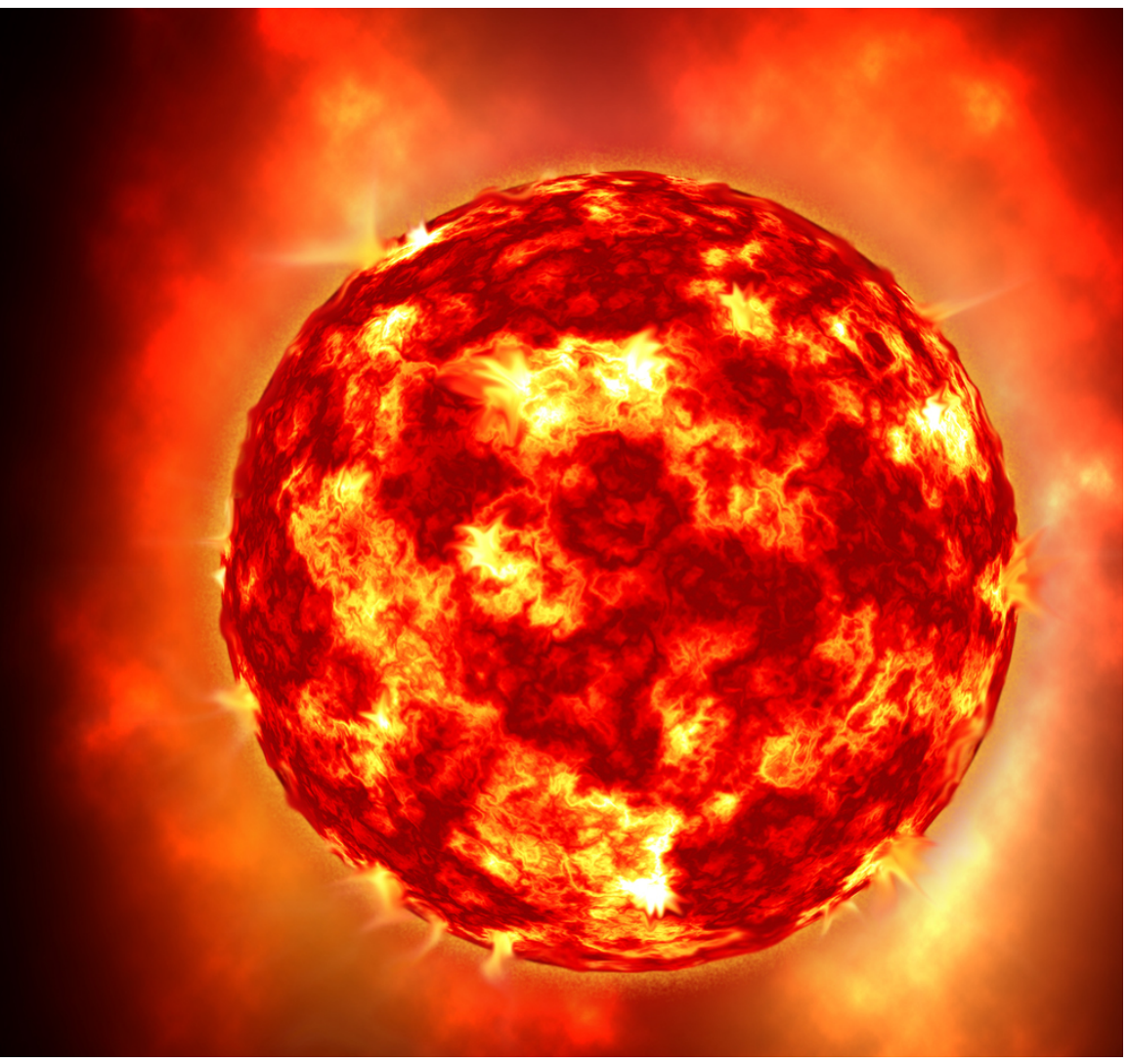

nes de años. Sin embargo, cálculos detallados mostraban que dicho proceso no podía permitir al Sol brillar por más de unas pocas decenas de millones de años, en contradicción directa con las conclusiones de geólogos y biólogos tales como dCharles Lyell y Charles Darwin, que habían mostrado que para explicar la estructura de la Tierra y la diversidad de las especies era necesario que el Sol hubiera brillado de manera más o menos constante por cientos o quizá miles de millones de años. Eddington observó que el único proceso capaz de proveer al Sol de energía por tiempos suficientemente largos era la conversión directa de materia en energía, postulada por Einstein en 1905 en su famosa ecuación $\mathrm{E}=\mathrm{mc}^{2}$. Eddington sabía también que unos años antes se había mostrado que la masa de un átomo de helio era aproximadamente $1 \%$ menor que la masa de cuatro átomos de hidrógeno, y razonó que, si cuatro átomos de hidrógeno pudieran combinarse para formar un átomo de helio en el interior de Sol, la energía liberada en el proceso sería más que suficiente para explicar el brillo del Sol y las estrellas por miles de millones de años. Éste fue el origen de la idea de que en el interior de las estrellas elementos ligeros se "fusionan" para formar elementos más pesados, liberando enormes cantidades de energía.

Nuestra comprensión de la estructura del núcleo atómico tuvo que esperar hasta el descubrimiento de los neutrones en 1932 por parte de James Chadwick, partículas de masa muy similar a la del protón, pero sin carga eléctrica. El núcleo atómico no estaba formado solamente por protones de carga positiva, sino también por neutrones sin carga eléctrica. Para entonces quedaba claro que los distintos elementos diferían sólo en el número de protones en el núcleo, y que sus propiedades químicas estaban determinadas por los electrones que los orbitaban. Ese mismo año, Carl David Anderson realizó otro descubrimiento fundamental, el positrón o anti-electrón, una partícula idéntica al electrón, pero de carga eléctrica opuesta. Dicha partícula había sido predicha por Paul Dirac en 1928, y constituía la primera evidencia de la existencia de lo que sería llamado la anti-materia.

Hacia fines de la década de 1930, Lise Meitner y Otto Hahn habían logrado demostrar que era posible transmutar un elemento en otro bombardeando los núcleos atómicos con neutrones que ocasionaban que dichos núcleos se fragmentaran: se había descubierto la "fisión" nuclear. ' La fisión de núcleos pesados

Nobel de física en 1944 por el descubrimiento de la fisión nuclear. El que el premio no haya sido también otorgado a Lise Meitner es una de las mayores controversias en la historia de los premios Nobel. 
2. Hans Bethe recibió el premio Nobel de física en 1967 por estos estudios. liberaba cantidades importantes de energía, hecho que quedó de manifiesto con la creación de los primeros reactores nucleares y las bombas atómicas pocos años después.

El descubrimiento del neutrón y el positrón permitía también explicar la fusión nuclear del hidrógeno en helio propuesta por Eddington. Al fusionarse cuatro núcleos de hidrógeno se debería tener un núcleo de carga positiva igual a cuatro, mientras que la carga del núcleo de helio es de sólo dos. Pero si en el proceso dos de los protones se transformaban en neutrones, liberando la carga eléctrica sobrante en forma de dos positrones, el resultado sería precisamente un núcleo de helio.

La idea original de Eddington quedó firmemente establecida en 1939, cuando Hans Bethe analizó en detalle las distintas reacciones nucleares que podían tener lugar en el interior de las estrellas para convertir hidrógeno en helio. ${ }^{2}$ La fusión de hidrógeno en helio era en efecto posible, y liberaba cantidades de energía mucho mayores que la fisión de núcleos pesados, como quedó dramáticamente demostrado con el desarrollo de las bombas de hidrógeno, devastadores soles en miniatura.

Bethe no consideró la formación de núcleos aún más pesados, pero en 1946 Fred Hoyle mostró cómo a muy altas temperaturas era posible fusionar átomos de helio para formar nitrógeno, carbono y oxígeno, y continuar así una cadena de fusiones nucleares hasta el hierro. El hierro, sin embargo, es un callejón sin salida: mientras que la fusión de elementos ligeros con masas menores a las del hierro libera energía, la fusión del hierro y elementos más pesados absorbe energía. En otras palabras, es imposible generar elementos más pesados que el hierro en el interior de las estrellas.

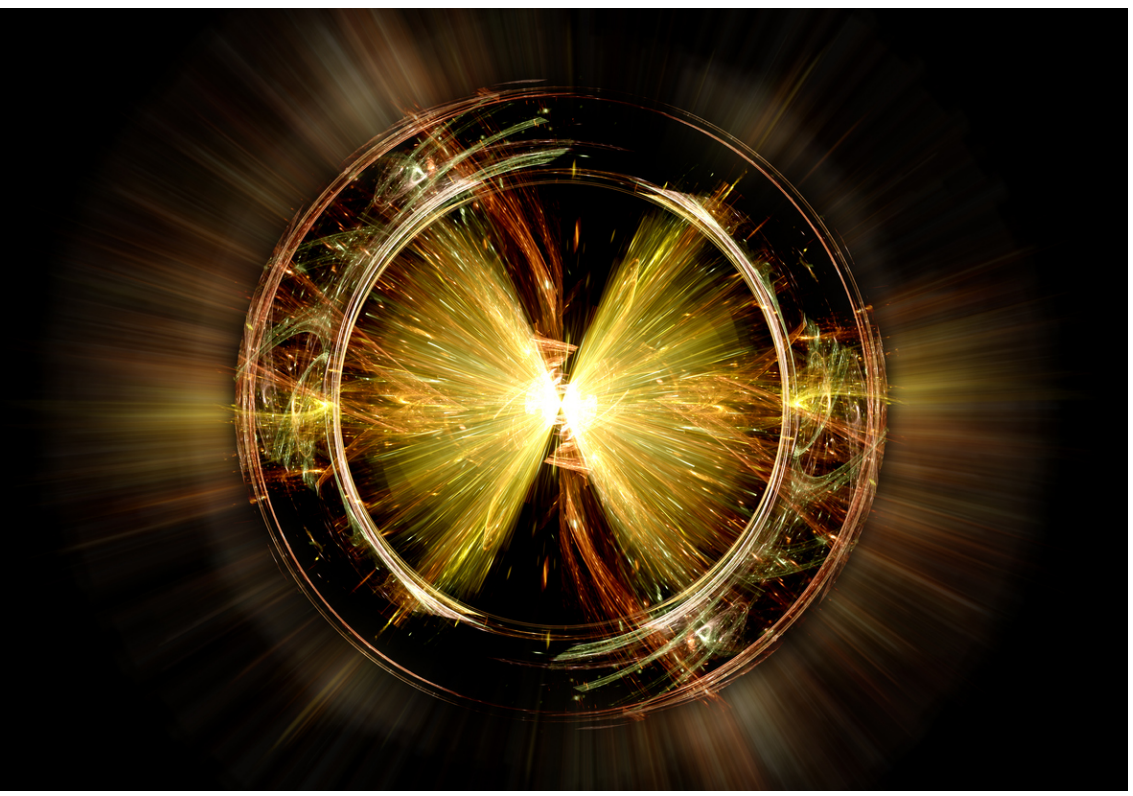

Para la década de los setenta, la teoría básica de la formación de elementos en el interior de las estrellas, la llamada "nucleosíntesis estelar", estaba completa. Las estrellas como el Sol, Ilamadas de secuencia principal, obtienen su energía de fusionar hidrógeno en helio en su interior. El helio se acumula lentamente en el centro hasta que la temperatura se eleva lo suficiente como para que el helio se fusione en elementos aún más pesados. A partir de ese momento el proceso se acelera, y se producen sucesivamente carbono, nitrógeno, oxígeno, neón y elementos cada vez más pesados hasta llegar al hierro. El hierro finalmente se acumula en el centro sin poder fusionarse y eventualmente la estrella se apaga y muere. 
El origen de los elementos hasta el hierro quedaba entonces claro, pero existían aún dos incógnitas: ¿cómo se forman los elementos más pesados que el hierro?, y ¿cómo salen los elementos del interior de las estrellas para formar planetas, lunas y vida?

La respuesta a ambas preguntas tiene que ver con la forma en la que las estrellas mueren. Estrellas poco masivas como el Sol mueren cuando se acumula carbono en el centro, lo que ocasiona que se apaguen y se enfríen poco a poco ya que, por su baja masa, no alcanzaron temperaturas suficientemente altas para producir la fusión del carbono en elementos más pesados.

Pero estrellas masivas, de diez o más masas solares, sufren un fin mucho más espectacular. Al apagarse las reacciones nucleares en el centro, las capas externas de la estrella comienzan a caer sobre el núcleo de forma violenta, provocando un nuevo incremento catastrófico de la temperatura, que produce una explosión final que expulsa todas las capas externas al espacio exterior: una supernova.

La explosión de la supernova explica cómo escapan los elementos formados en el interior de las estrellas hacia el exterior. Pero también puede explicar la formación de elementos aún más pesados que el hierro, como sugirió el mismo Fred Hoyle en 1954. Durante la explosión se alcanzan temperaturas suficientemente elevadas como para fusionar núcleos de hierro y aún más allá, permitiendo la formación de elementos mucho más pesados que serán dispersados por el espacio. Sin embargo, aunque dicha explicación parece funcionar en principio, cálculos detallados muestran que no puede explicar las abundancias observadas de elementos pesados. Había algo que aún no quedaba claro.

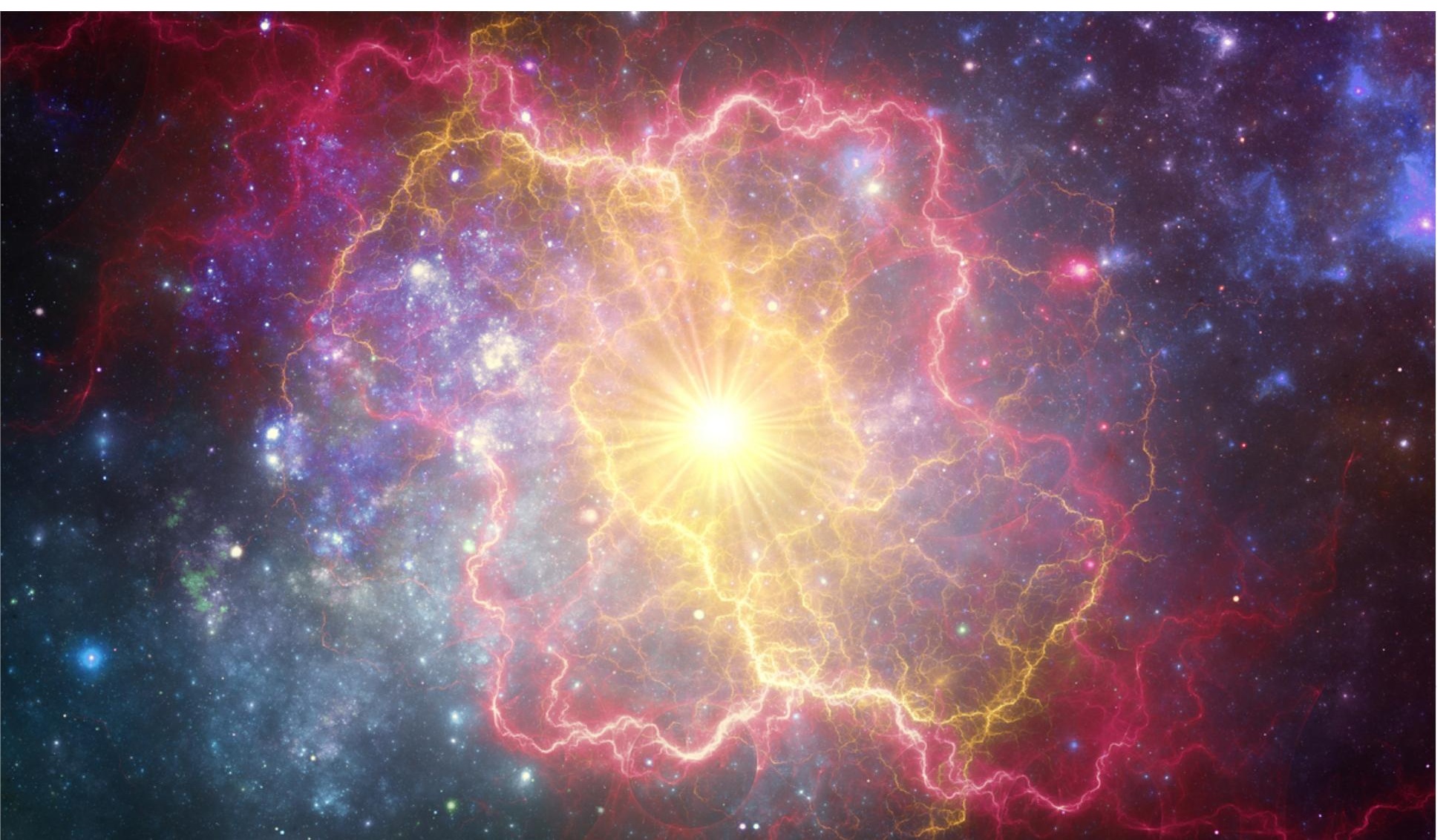




\section{Estrellas de neutrones y púlsares binarios}

La historia del origen de los elementos debe ahora tomar otra dirección que nos lleva al estudio de objetos astrofísicos exóticos llamados estrellas de neutrones. La historia de estas estrellas se remonta a 1930, cuando el físico indio Subrahmanyan Chandrasekhar estudió la estructura de estrellas utilizando la teoría de la relatividad de Einstein y los principios de la mecánica cuántica.

Como mencionamos anteriormente, las estrellas como el Sol mueren cuando se acumula hierro en su centro y se apagan las reacciones nucleares, enfriándose y contrayéndose poco a poco hasta convertirse en enanas blancas. Pero si las enanas blancas están frías, ¿qué es lo que las sostiene y evita que se contraigan aún más?

Chandrasekhar concluyó que las enanas blancas se sostienen por un tipo de presión que tiene su origen en la mecánica cántica, y en particular en el principio de exclusión de Pauli, que dice que dos electrones no pueden estar en el mismo estado cuántico. En una enana blanca, la densidad es tal alta que los electrones están prácticamente tocándose unos a otros, y esto produce una presión debida al principio de Pauli que sostiene a la estrella aún si su temperatura es muy baja. Pero Chandrasekhar fue aún más lejos, y mostró que hay una masa máxima más allá de la

3. Chandrasekar recibió el premio Nobel en 1983 por su trabajo en la estructura y evolución de las estrellas. cuál esta presión es insuficiente y la estrella no puede mantenerse en equilibrio. Esta masa máxima, conocida como el límite de Chandrasekhar, es de aproximadamente 1.4 veces la masa del Sol. ${ }^{3}$ En 1930 no se conocía ninguna manera de detener el colapso y se suponía que una enana blanca que rebasara esta masa se continuaría colapsando hasta concentrarse en un solo punto, creando lo que hoy en día conocemos como un agujero negro.

Pero el descubrimiento de los neutrones en 1932 cambió el panorama. En 1934 Walter Baade y Fritz Zwicky propusieron que, al colapsarse una enana blanca la alta presión forzaría a los electrones a fusionarse con los protones, produciendo neutrones. Se tendría entonces una estrella compacta formada exclusivamente por neutrones. Baade y Zwicky propusieron también que la 
4. El descubrimiento de los púlsares fue motivo para otorgar el premio Nobel de física en 1974 a Antony Hewish. El que el premio no se haya otorgado también a Jocelyn Bell, quien era estudiante de Hewish en 1967 y fue la primera en observar la emisión del pulsar, recuerda de cerca el caso de Lise Meitner. formación violenta de un núcleo de neutrones durante la muerte de una estrella muy masiva liberaría suficiente energía como para producir una supernova, dejando como resultado una estrella de neutrones en su centro. El estudio teórico mostró qué de existir estos objetos, la conservación de la cantidad de movimiento rotacional o momento angular, los obligaría a girar a altas velocidades, decenas de veces por segundo.

Las estrellas de neutrones fueron finalmente descubiertas en 1967, cuando Jocelyn Bell y Antony Hewish descubrieron un objeto astronómico que producía pulsos de radio de manera regular, al que se denominó "pulsar". ${ }^{4}$ Estudios posteriores permitieron identificar este pulsar, y los muchos que se han descubierto desde entonces, como estrellas de neutrones que giran a grandes velocidades y emiten chorros de radiación electromagnética por sus polos.

En 1974, Joseph Taylor y Russel Hulse descubrieron el primer "pulsar binario", una pareja de estrellas de neutrones en órbita, una alrededor de la otra. Los pulsares binarios forman otra de las piezas clave en el estudio del origen de los elementos, como veremos más adelante.

Las estrellas de neutrones también tienen su propio límite de Chandrasekhar, la masa máxima más allá de la cual no pueden sostenerse. Pero a diferencia del caso de las enanas blancas donde la física se entiende en gran detalle, a la fecha no se conoce con precisión la masa máxima de una estrella de neutrones debido a la complejidad de estudiar la interacción de neutrones a altas presiones. Las estimaciones más recientes sitúan este límite entre dos y tres veces la masa del Sol.

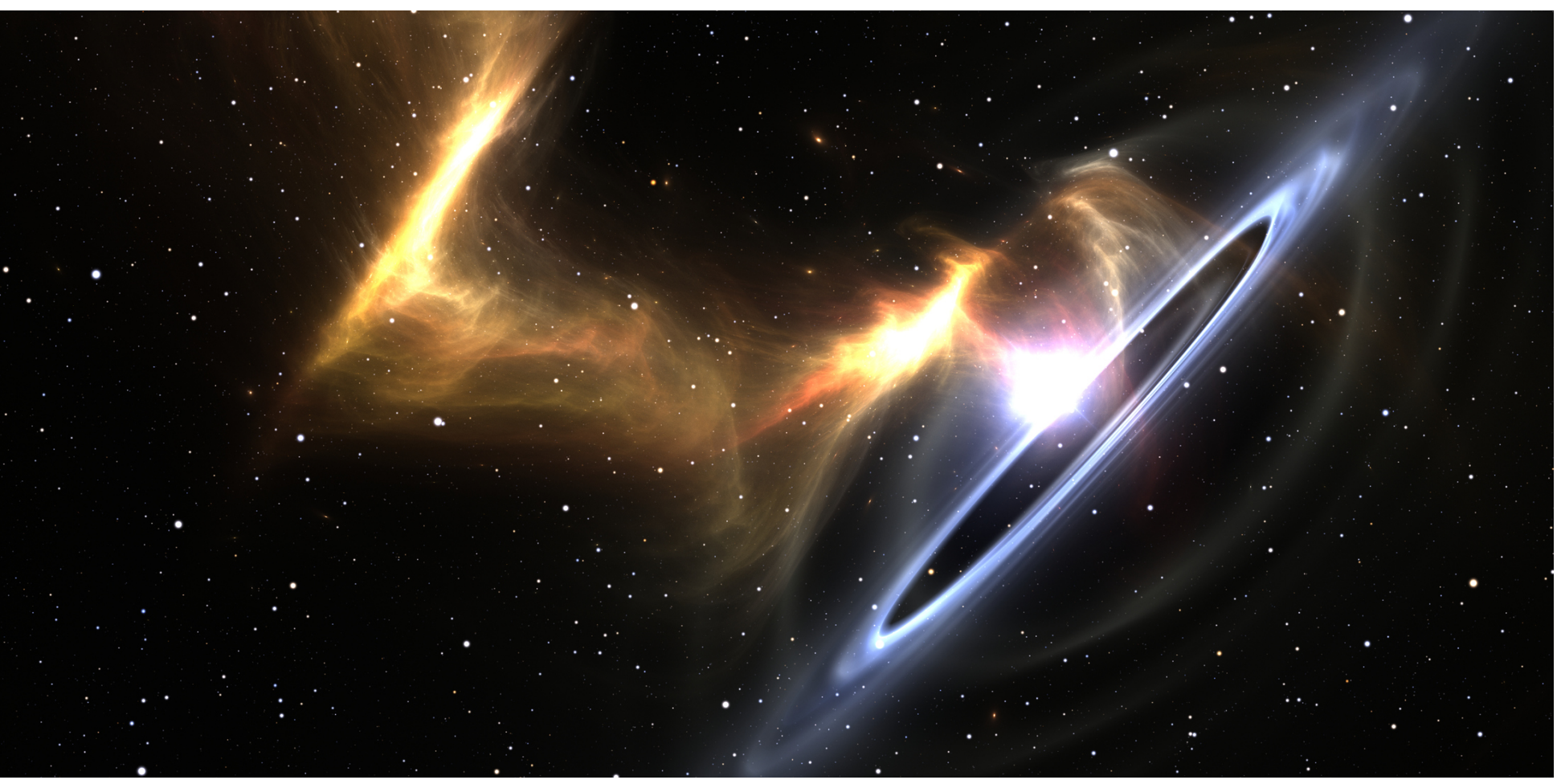


¿Y más allá de las estrellas de neutrones? Hasta donde entendemos hoy en día, más allá de las estrellas de neutrones ya no existe ningún estado estable: una estrella de neutrones que supera su masa máxima colapsará inevitablemente hasta formar un agujero negro. Aunque existen propuestas especulativas sobre un último estado anterior al agujero negro llamado "estrella de quarks", a la fecha no es claro si este tipo de objetos puede existir en realidad. Y aún en caso de que lo hiciera, la masa máxima para este tipo de objetos no sería mucho mayor, las estrellas muy masivas inevitablemente se convierten en un agujero negro al final de su vida.

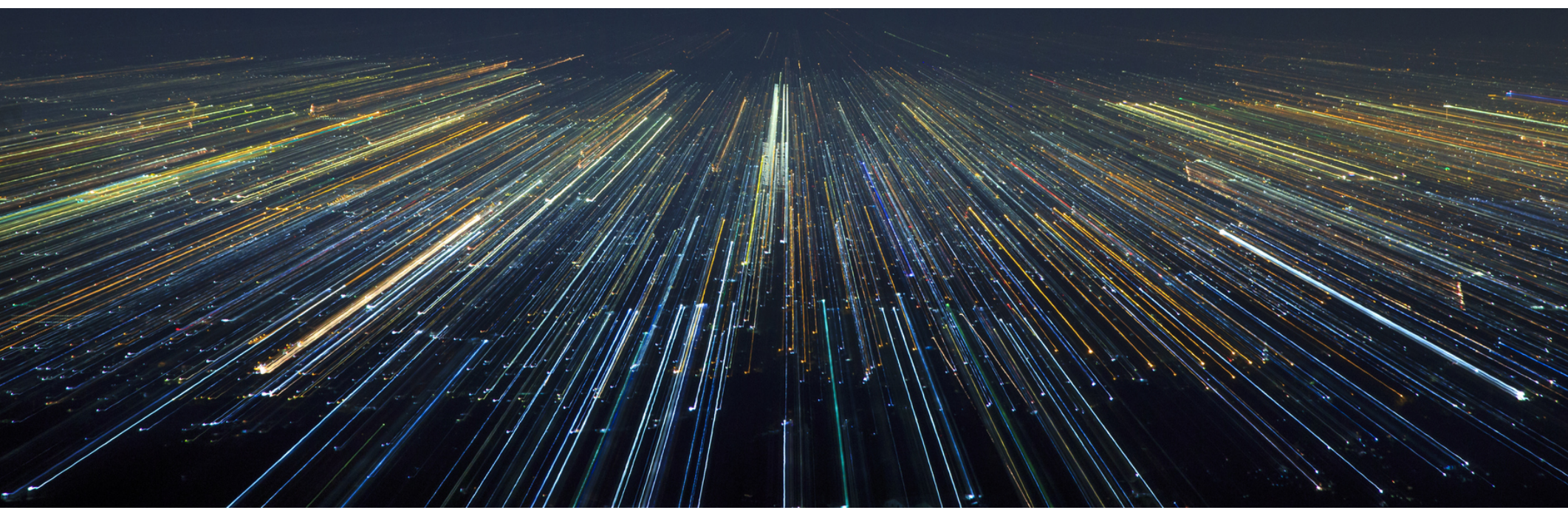

\section{Las ondas gravitacionales}

En 1905 Albert Einstein revolucionó nuestros conceptos de espacio y tiempo al desarrollar la teoría especial de la relatividad. Entre sus muchas consecuencias se encontraba el hecho de que no sólo la velocidad de la luz resulta ser absoluta, sino que además representa un límite máximo en la velocidad de propagación de cualquier fenómeno físico. Nada, ningún objeto, ninguna señal, ni ninguna interacción física de ningún tipo puede moverse más rápido que la luz. Este hecho entraba en claro conflicto con la teoría de la gravitación universal de Newton, según la cual la gravedad actuaba de manera instantánea. Era claro que había llegado el momento de modificar la teoría de la gravedad, y Einstein dedicó los siguientes diez años a esta tarea. El resultado final de sus esfuerzos fue la teoría general de la relatividad de 1915, una nueva teoría de la gravitación totalmente compatible con los conceptos relativistas de espacio y tiempo. De acuerdo con la relatividad general la gravedad no se propaga de manera instantánea, sino que lo hace precisamente a la velocidad de la luz. Y, de la misma forma que el electromagnetismo predice la existencia de ondas electromagnéticas, la relatividad general predice la existencia de ondas gravitacionales, perturbaciones de la gravedad que se propagan a la velocidad de la luz. 
5. Hulse y Taylor recibieron el premio Nobel en 1993 por este trabajo.
Sin embargo, pese a haber sido predichas por Einstein en 1916, las ondas gravitacionales resultaron sumamente difíciles de detectar. La primera evidencia indirecta de su existencia llegó en 1974 con el descubrimiento del pulsar binario de Hulse y Taylor. De acuerdo con la teoría de Einstein, cuando se tiene un sistema de objetos compactos muy masivos en órbita uno alrededor de otro, el sistema debe emitir ondas gravitacionales y como consecuencia perder energía, por lo que los objetos se irán acercando poco a poco. El pulsar binario resultaba un sistema ideal para estudiar este fenómeno. Durante los veinte años siguientes a su descubrimiento, se estudió detalladamente la órbita de ambas estrellas y se notaron cambios en la velocidad orbital que correspondían precisamente con los que predecía la relatividad general como consecuencia de la emisión de ondas gravitacionales. ${ }^{5}$

La detección directa de las ondas gravitacionales debió esperar aún varias décadas. El problema principal es que la gravedad es una fuerza extremadamente débil. Cálculos teóricos muestran que la explosión de una supernova en nuestra galaxia, o el choque de dos estrellas de neutrones en una galaxia cercana, produciría ondas gravitacionales tales que al pasar por la Tierra causarían oscilaciones en los aparatos de medición de aproximadamente una parte en cien millones de billones (un uno seguido de veinte ceros). Es decir, un aparato de medición con una longitud equivalente al diámetro de la Tierra experimentaría una deformación equivalente al ta-

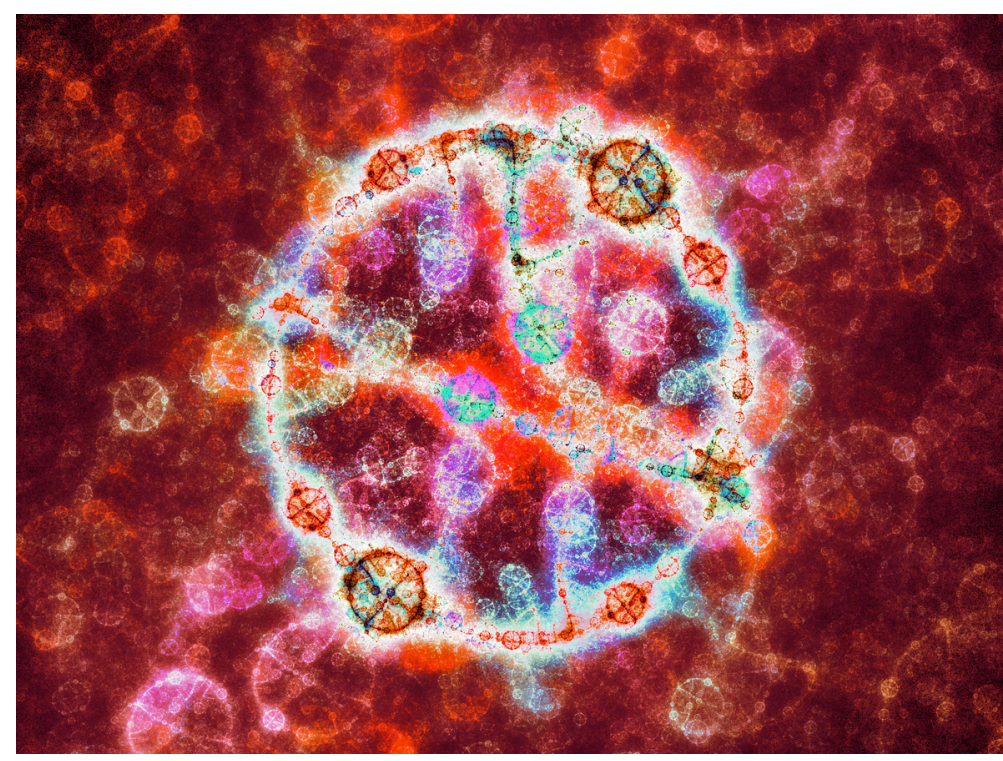
maño de un núcleo atómico. Medir algo tan pequeño suena tan manifiestamente absurdo que nadie lo intentó siquiera por varias décadas.

La primera persona que intentó detectar ondas gravitacionales fue Joseph Weber en la década de 1960. Para ello construyó dos barras de aluminio de varias toneladas de peso con la idea de medir las pequeñas oscilaciones producidas en ellas por el paso de una onda gravitacional. A mediados de 1969, Weber anunció que sus barras habían detectado ondas gravitacionales provenientes del centro de nuestra galaxia, y durante los siguientes años anunció aún más detecciones. El problema vino cuando grupos independientes de científicos intentaron reproducir las observaciones de Weber con sus propias barras y no pudieron detectar nada. Después de diez años en esta situación la comunidad internacional concluyó que Weber en realidad nunca había detectado ondas gravitacionales, aunque trágicamente Weber mismo continuó afirmándolo hasta su muerte. 


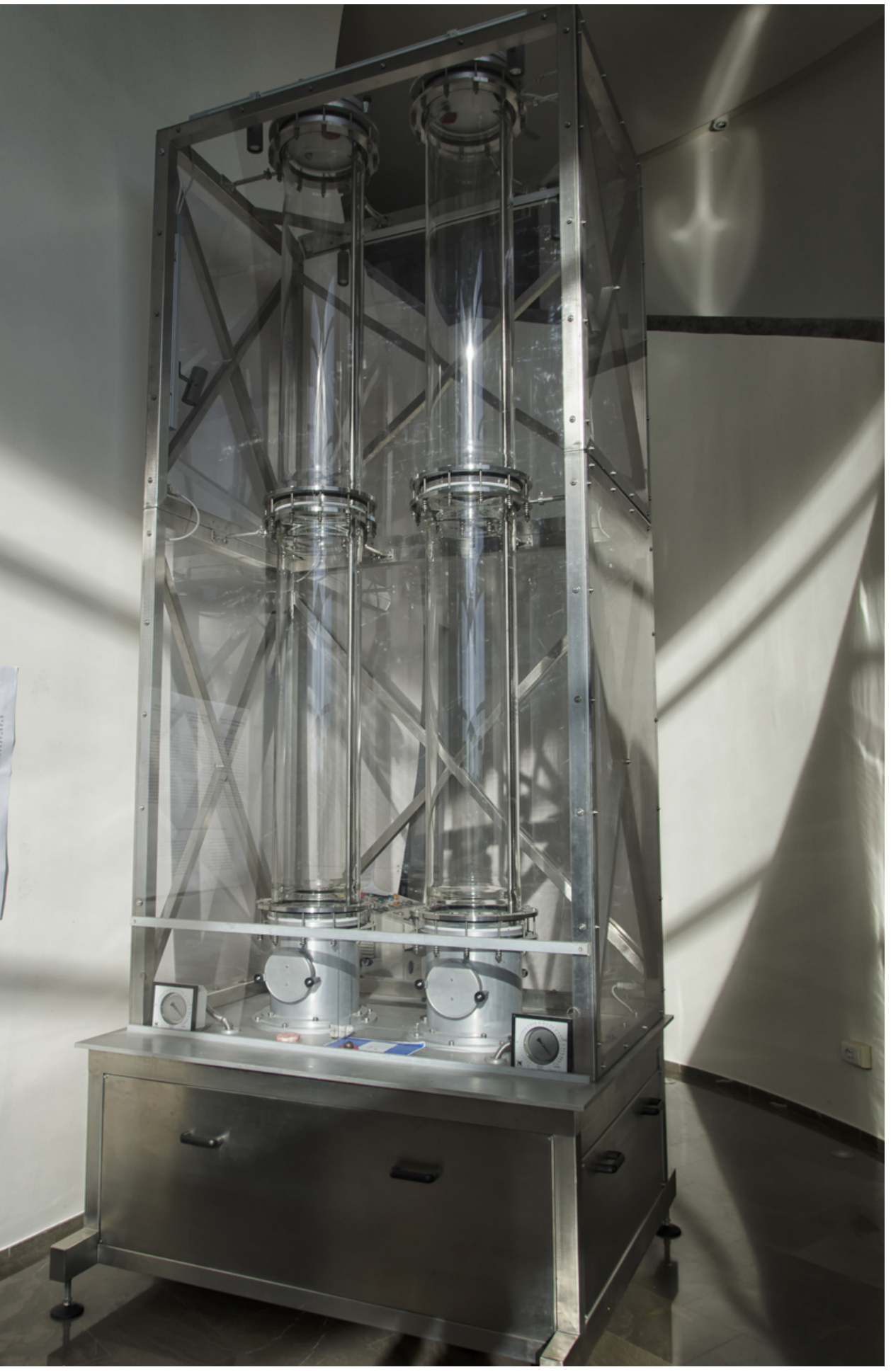

Para principios de los años ochenta varios grupos experimentales llegaron a la conclusión de que, si bien Weber no había detectado en realidad ondas gravitacionales, la idea de lograr detectarlas no era tan descabellada, y debería buscarse un método diferente que tuviera mayores posibilidades de detección. Para ello se concentraron en la idea de un aparato conocido como interferómetro, donde rayos láser recorren caminos perpendiculares hasta espejos, para luego regresar y combinarse. Debido a que la luz es una onda, al recombinarse los rayos se produce interferencia, y ésta puede utilizarse para detectar con altísima precisión pequeños cambios en las posiciones de los espejos producidos ya sea por vibraciones del suelo, o por ondas gravitacionales provenientes del espacio.

En Estados Unidos se estableció a principios de los noventa el proyecto del "Observatorio Gravitacional por Interferometría Láser", o LIGO por sus siglas en inglés, que consistía en dos interferómetros de cuatro kilómetros de largo cada uno, colocados a 3000 kilómetros de distancia uno del otro. Un proyecto similar se creó en Italia, el proyecto viRGo, y uno más pequeño en Alemania.

Después de más de quince años de construcción y pruebas, el proyecto LIGo dio frutos en septiembre de 2015 cuando ocurrió la primera detección confirmada de ondas gravitacionales, provenientes de la colisión de dos agujeros negros, con masas de aproximadamente 29 y 36 masas solares respectivamente, a una distancia de unos 1,300 millones de años luz de la Tierra. De entonces a la fecha se han detectado otros cuatro eventos correspondientes a la colisión de agujeros negros, el último de los cuales también fue detectado por el observatorio italiano viRGo. 
6. Una nova es un evento astronómico transitorio causado por el intercambio de materia entre dos estrellas cercanas que causa que una de ellas produzca una gigantesca erupción nuclear que aumenta su brillo de manera espectacular. No se debe confundir con una supernova que es la muerte de una estrella en una explosión catastrófica y puede ser un millón de veces más luminosa.
La detección directa de las ondas gravitacionales dio lugar a que el premio Nobel de física 2017 fuera otorgado a los proponentes iniciales de LIGO, Rainer Weiss y Kip Thorne, junto con Barry Barish, quien fuera el primer director del proyecto.

\section{La kilonova GW170817}

A partir de las detecciones de LIGo la realidad física de las ondas gravitacionales quedó firmemente establecida, y se dio inicio a una nueva rama de la astronomía, la astronomía de ondas gravitacionales.

Pero la detección más espectacular se dio el 17 de agosto de 2017, cuando por primera vez se detectó una señal en ondas gravitacionales en coincidencia con un destello de rayos gamma. La presencia de los rayos gamma indicaba que este evento ya no correspondía al choque de dos agujeros negros, sino al choque de dos estrellas de neutrones a una distancia de 130 millones de años luz de la Tierra. Dicho evento fue posteriormente observado en luz visible por un observatorio en Chile, lo que permitió determinar la galaxia de origen, y fue seguido por decenas de observatorios, tanto espaciales como terrestres, en todo el espectro electromagnético: rayos gamma, rayos $X$, luz visible, luz infrarroja y ondas de radio. El evento fue bautizado como una "kilonova" por involucrar energías unas mil veces mayores que una nova. ${ }^{6}$

La detección de esta kilonova ha dado inicio a una nueva era de la astronomía, donde un mismo evento es observado de muchas maneras distintas por muchos observatorios diferentes. En este caso participaron más de setenta observatorios alrededor de todo el mundo, y más de 4000 astrónomos... la astronomía multi-mensajero ha comenzado.

Pero mucho más interesante para la historia del origen de los elementos es el hecho de que, en las observaciones posteriores al evento, fue posible detectar la señal de la presencia de elementos más pesados que el hierro, y en particular metales pesados como el oro y el platino en gran abundancia. La kilonova produjo, a partir de dos estrellas de neutrones no mucho más masivas que el Sol, varios miles de veces la masa de la Tierra en elementos pesados, y al menos diez veces la masa de la Tierra en oro y platino.

La abundancia de elementos pesados indica que las colisiones de estrellas de neutrones pueden ser el elemento faltante en la formación de los elementos pesados, y su dispersión por el Universo, para formar a partir de ellos rocas, planetas y lunas. 


\section{Conclusión}

Los desarrollos científicos de la primera mitad del siglo xx nos permitieron entender la estructura de los átomos y sus núcleos, y nos mostraron que es posible transformar unos elementos en otros, ya sea fisionando núcleos pesados, o fusionando núcleos ligeros. El origen de los elementos más pesados que el hidrógeno y el helio está precisamente en los procesos de fusión nuclear que ocurren en el interior de las estrellas y que a la vez proporcionan la energía necesaria para que estas brillen por miles de millones de años. Sin embargo, elementos más pesados que el hierro no pueden formarse de esta manera, pues la fusión del hierro es un proceso que absorbe energía en lugar de liberarla. La formación de elementos aún más pesados requiere de procesos catastróficos, como la explosión de una supernova o el choque de dos estrellas.

La reciente detección de las ondas gravitacionales ha abierto una nueva ventana al Universo. La observación en agosto de 2017 de las ondas gravitacionales provenientes de la colisión de dos estrellas de neutrones, en coincidencia con un destello de rayos gamma, nos ha permitido por primera vez estudiar este tipo de eventos con alta precisión. Dicho evento, también conocido como una kilonova, fue observado por más de setenta observatorios en todo el mundo, en todo el espectro electromagnético, dando inicio a la llamada astronomía de mensajeros múltiples.

La observación detallada de la kilonova durante las semanas siguientes a la colisión nos permitió mostrar qué dio lugar a la formación de grandes cantidades de elementos pesados, y en particular metales preciosos como el oro y el platino. Podemos, entonces, imaginar que el oro que existe en la Tierra se formó hace miles de millones de años, en colisiones de estrellas de neutrones que dispersaron sus restos por la galaxia y sembraron las nubes de polvo de las que después de formaría el sistema solar.

\section{Cómo citar este artículo}

* Alcubierre Moya, Miguel (2018). El choque de estrellas de neutrones y la alquimia del Universo. Revista Digital Universitaria (RDU). Vol. 19, núm. 3 mayo-junio. DOI: http://doi.org/10.22201/codeic.16076079e.2018.v19n3.a3. 